\title{
Photoelectron-Pulse Properties from Free-Free Transitions in Ultrafast Transmission Electron Microscopy
}

\author{
David J. Flannigan
}

Department of Chemical Engineering and Materials Science, University of Minnesota, Minneapolis, MN, 55455, USA

In the presence of an intense laser pulse, electrons photoejected from a surface can be accelerated by the optical electric field [1]. This can occur because momentum is conserved via coupling with the structure, which results in the absorption and stimulated emission of photon quanta of energy by the photoejected electrons [2]. A similar process can occur during the precise spatiotemporal overlap of a freely-propagating relativistic electron with an optical field at a nanostructure. Maxwell's equations tell us that electric-field enhancement can occur at the surfaces of nanostructures illuminated with visible light. Certain nanostructure features (e.g., corners or edges) act to further enhance the electric field due to the spatial confinement of coherent electron oscillations known as plasmons. Relativistic electrons passing through such enhanced fields near a nanostructure can gain and lose photon quanta of energy (i.e., free-free transitions) similar to the photoejected geometry.

With ultrafast transmission electron microscopy (UTEM), access can be gained to the spatiotemporal scales required to directly visualize rapid, non-equilibrium structural dynamics of materials [3]. This is achieved by operating a transmission electron microscope (TEM) in a stroboscopic pump-probe fashion by photoelectrically generating coherent, precisely-timed electron packets. These probe photoelectrons are accelerated down the TEM column where they travel through the specimen before reaching a standard CCD detector. A second laser pulse is used to excite (pump) the specimen in situ. Structural changes are visualized by varying the arrival time of the pump laser pulse relative to the probe electron packet at the specimen. For physical processes occurring on timescales commensurate with the instrument response, deconvolution is critical to isolating the intrinsic dynamics so that a clear picture of the ultrafast mechanisms can be formed. In order to accomplish this, one must have a way to precisely measure the instrument response function at the specimen location.

Here, I will briefly review how free-free transitions of $200 \mathrm{keV}$ electrons can be induced and detected with UTEM equipped with an energy filter and spectrometer [4-6]. Following this, I will describe how the response of the low-loss region in the UTEM electron energy spectrum is caused by varying the temporal overlap of the femtosecond photon and electron pulses when spatially static and aligned at a nanostructure. Temporal variation of the pulses relative to one another causes a modulation of the partitioning of the $200 \mathrm{keV}$ electrons into quantized virtual states (Figure 1). This modulation can be detected in the low-loss region (up to $\sim 100 \mathrm{eV}$ ) of the electron energy spectrum. Variation in the partitioning causes the zero-loss peak intensity to fall and rise, with the curve being a convolution of the shapes of the two interacting pulses, thus revealing the overall instrument response function. In this way, intrinsic structural dynamics occurring on ultrafast timescales can be isolated from the overall instrument response. Further, this method provides a means to characterize the femtosecond electron pulse properties at the specimen location. In this way, one could perform systematic experimental studies of the effects of laser pulse properties, cathode work function, and lens settings (among other parameters) on the shape and duration of the probing electron pulse. Thus, this method provides for a practical approach to characterizing and optimizing femtosecond electron pulse properties in UTEM [7]. 
References:

[1] G. Saathoff, L. Miaja-Avila, M. Aeschlimann, M. M. Murnane and H. C. Kapteyn, Phys. Rev. A 77 (2008), 022903.

[2] A. Weingartshofer, J. K. Holmes, G. Caudle, E. M. Clarke and H. Krüger, Phys. Rev. Lett. 39 (1977), 269.

[3] D. J. Flannigan and A. H. Zewail, Acc. Chem. Res. 45 (2012), 1828.

[4] B. Barwick, D. J. Flannigan and A. H. Zewail, Nature 462 (2009), 902.

[5] D. J. Flannigan, B. Barwick and A. H. Zewail, Proc. Natl. Acad. Sci., U.S.A. 107 (2010), 9933.

[6] S. T. Park, M. Lin and A. H. Zewail, New J. Phys. 12 (2010), 123028.

[7] All experimental work was performed at the California Institute of Technology in the labs of Prof. Ahmed H. Zewail. The original results from the experimental work were reported in [4], with subsequent experiments on soft-matter and biological systems reported in [5].
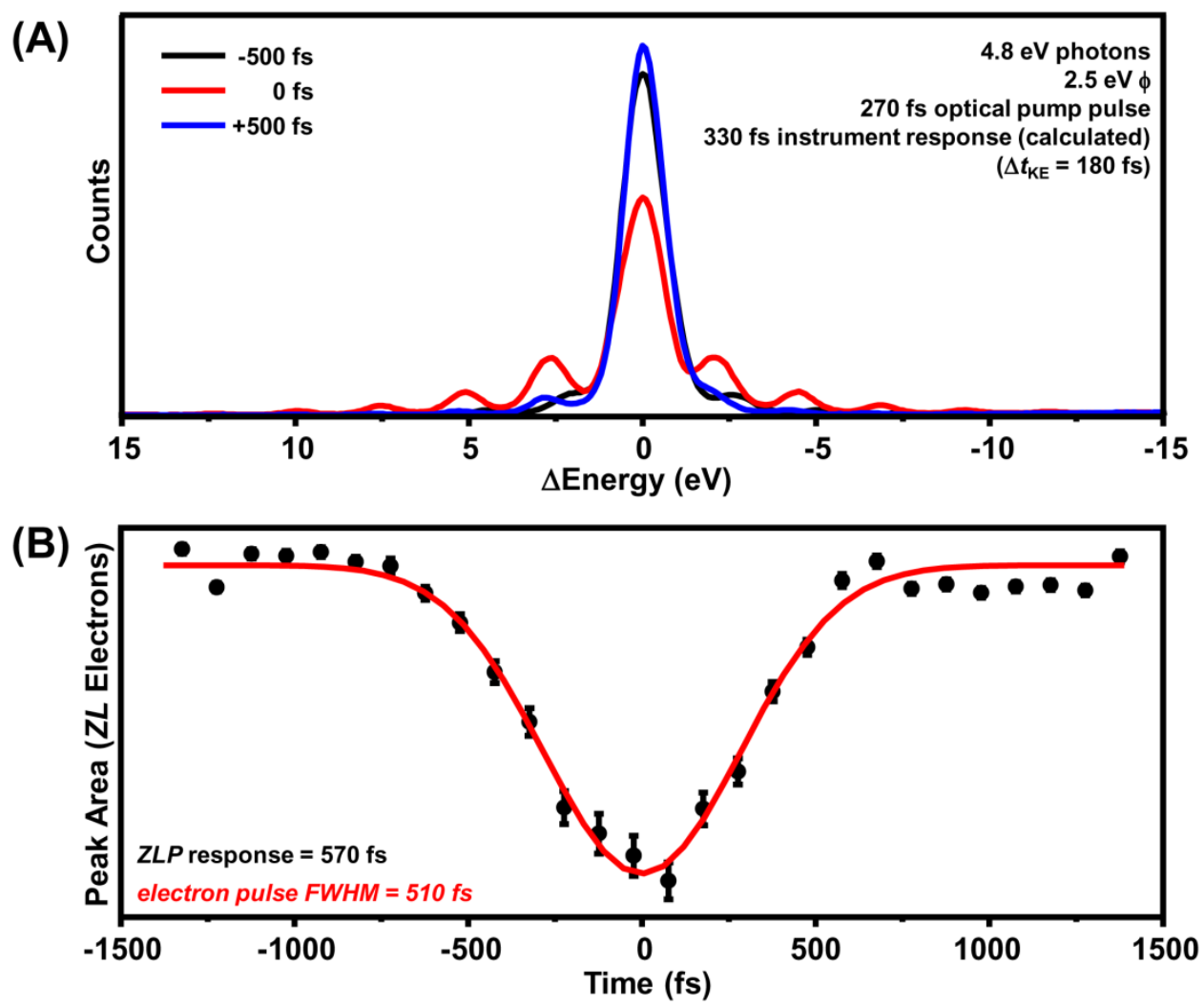

Figure 1. Time-dependent low-loss energy spectra (A) and zero-loss peak area (B) for spatiallyoverlapped temporally-scanned femtosecond photon and electron pulses in UTEM [5,7]. In (A), spectra are shown for cases when the pulses were precisely overlapped $(0 \mathrm{fs})$ and offset relative to one another by $\pm 500 \mathrm{fs}$. For the experimental parameters shown, the calculated instrument response is $330 \mathrm{fs}$ (spacecharge-free regime), with a contribution of 180 fs due to the photon energy/work function mismatch. In (B), the temporal dependence of the zero-loss peak area (i.e., the total instrument response) is shown with a Gaussian least-squares fit. Deconvolution of the Gaussian optical pump pulse returns an electron pulse FWHM of $510 \mathrm{fs}$. Error bars are two-sigma and were determined from the quality of the fit to the zero-loss peak. Experiments were performed at the California Institute of Technology in the labs of Prof. Ahmed H. Zewail [5]. 